\title{
Is the Contested Urban Networked Littoral Environment a Step too far for Agent-Based-Modelling?
}

\author{
W.T. Johnson ${ }^{\mathrm{a}}$ and V.G. Ivancevic ${ }^{\mathrm{b}}$ \\ ${ }^{\mathrm{a}}$ Land Division (LD), Defence Science \& Technology Group (DST Group), GPO BOX 1500, Edinburgh, \\ Australia, 5111. \\ ${ }^{\mathrm{b}}$ Joint Operations and Analysis Division (JOAD), DST Group, Australia \\ Email: wayne.johnson@defence.gov.au
}

\begin{abstract}
The broad Defence problem addressed by our current work is, how do we handle the expansion of our modelling requirements to deal with violent conflict in contested urban environments (CUE)? Guidance regarding the relevance of the CUE from the upper echelons of Defence with Chief of Army endorsement, can be found in the Modernisation and Strategic Planning Division Staff's Future Land Warfare Report (FLWR) (MSPD-Staff, 2014), and in Kilcullen's (2014) “The Australian Army in the Urban Networked Littoral”. Both echo a similar message that future military operations are most likely to be held in highly networked littoral CUE, where the degree of complexity of such environments is far beyond that of conventional force-on-force engagement.
\end{abstract}

With regard to the operating environment FLWR (MSPD-Staff, 2014) indicates that the environment for future operations has five meta-trends; crowded, connected, lethal, collective, constrained. The term 'crowded' encompasses a range of factors that interplay to create complex human, informational and urban physical terrain, including urbanisation, rural to urban migration, population growth, resource scarcity and environmental and regional political instability. The term 'connected' refers to the flattening effect of the interconnected global economic, social and communications systems. It is Kilcullen's (2014) seminal observation, that the central novel feature is the highly networked nature of the urban world, which encompasses complex flow systems of trade, people, idea-systems, information with competition for not only territory, consisting of the natural land and built environs, but the built environment services such as, water, electricity, transportation, communication and the like. The competition does not, however, stop there; it is also a competition for idea-systems in what some have called the "battle for ideas" (Clarke, 2004). Many interrelated factors contribute toward population movements toward littoral mega-cities, upon whose physical terrain, exist turbulent seas of complex flowing dynamic topologies which we term the human-terrain.

This discussion paper attempts to start an exploration of the issues involved in studying violent conflict in the uncertain complex $C U E$. A small multi-disciplinary-team has been formed within LD and JOAD with an initial focus on the Knowledge Representation (KR) of littoral urban environments for highly networked actors within an Agent Based Model (ABM) and other simulations. We suggest, based on previous work of Johnson and Ivancevic $(2013 ; 2015)$, two psychological constructs which may well be applicable to ABM for either beauty of KR or computational efficiency.

The current work is being conducted as a horizon survey within the frame of unbounded systems thinking (UST) due to Mitroff and Linstone (1993). With regard to world views, in addition to the traditional perspective of science/technology, the Technical $T$ perspective, two other perspectives are taken into account in deciding what action to take next. They are the Organisational $O$ perspective of the social-entity and the Personal $P$ perspective of the self/individuation. It is a challenging approach in which everything is connected to everything and none of the sciences or professions involved in a study is considered fundamental.

Against this frame the sociological stance is considered at some length and seven basic aspects of sociological theory are introduced (Macionis and Gerber, 2010); culture, society, socialisation, social interaction in everyday life, groups and organisations, sexuality and society and finally deviance. We recommend that in highly networked urban environment that the sociology discipline be considered for modelling social-interactions, including emotions and language, within and between various social-groups for studies of future military operations within CUE. Finally, some difficult questions, from a UST point of view, are raised for using ABMs with respect to making progress towards defence outcomes.

Keywords: Agent-based-model, contested urban environment, unbounded systems thinking 
Is the Contested Urban Networked Littoral Environment a Step too far for Agent-Based-Modelling...

\section{INTRODUCTION}

The broad Defence problem addressed by our current work is, how do we handle the expansion of our modelling requirements to deal with violent conflict in contested urban environments (CUE)? At first blush it might appear that we can just rattle off a list of the obvious, those things that surely all can just see and just agree upon as the plain and simple single absolute truth of the matter. For instance, perhaps we could forthrightly just say that the new general requirements include the ability to model the following: a) civilians, non-stateactors, improvised explosive devices (IED), terrorism, asymmetric warfare; b) crowd behaviour dynamics and possible intervention/influence strategies on various scales; c) influence of belief-based ideological systems (Clarke, 2004), transmission of ideas and information, social influence; and d) cognitive modelling of actors in the environment? Perhaps? For the moment though, we pause to take in what our military have to say on the matter.

\subsection{Military guidance}

Guidance regarding the relevance of the CUE from the upper echelons of Defence, with Chief of Army endorsement, can be found in the Modernisation and Strategic Planning Division Staff's FLWR (MSPD-Staff, 2014), and in Kilcullen's (2014) “The Australian Army in the Urban Networked Littoral”. Both echo a similar message that future military operations are most likely to be held in highly networked littoral CUE ${ }^{1}$, where the degree of complexity of such environments is far beyond that of conventional force-on-force engagement.

The Australian army in the urban networked littoral. It is Kilcullen's (2014) seminal observation that the highly networked nature of the world is the new feature, not the urban or littoral aspects. This is in both degree and just what flows are networked, which increases both the complexity and uncertainty of the environment ${ }^{1}$. Networking encompasses complex flow systems of people, money, information with competition for not only territory, i.e. the natural land environment itself, but for built environment services such as, water, electricity, transportation, communication and the like ${ }^{2}$.

For instance, the degree of communications networking accelerates the existing trend of globalisation. It is this degree that is the main catalyst of change. As a specific example, Kilcullen (2014) reports a 279,000\% increase of mobile phone use in Nigeria over 10 years with almost as many phones as people. In turn this has created a diaspora by driving ethnic populations from the hinterlands to coastal centres, in the hunt for communications connectivity in order to participate in a global economy. This, says Kilcullen (2014), is the trend toward creating even more mega cities, and to such an extent that many littoral regions may become an endless city. We need to understand the cities and mega cities as integrated flow systems identifying not only the critical exchange points, but who in the populace controls the flows and to whose advantage. Just how violence, chaos and conflict can disrupt flows and shape cities, also needs to be understood. Fundamentally these urban topologies are highly dynamic and interrelated in nature, as opposed to the comparatively static natural physical terrain of conventional warfare.

Summary. The highest levels of the military upper echelons continually stress the importance of the human element, whether that be in support of the Australian digger at the coal face of operations (Sengelman, 2014), or of the generals in overall command having to demonstrate intellectual endeavour, boldness in decision making and compassion for their own troops, the enemy and civilians accidentally caught up in the action (Day, 2015). A critical part of the active dynamic flowing networked abstract-landscape or topology that is called the environment is the human-element, which includes our forces when deployed within $\mathrm{it}^{3}$.

\subsection{Uncertain complex dynamic Contested Urban Environments}

The dominant factor in the dynamic CUE is a rather pesky thing; the people who live in it, and perhaps are fighting in it. We call this dominant factor in the overall environment, the human terrain.

One aspect of dealing with the analysis of any military problem, whether it be in the context of actual operations or scientific support, is the importance of using an appropriate terrain model. No one would dare analyse a scenario without the correct geographic elevation landscape, i.e. the physical terrain. In order to model urban environments with their highly networked flows, as Kilcullen (2014) states, more than a model of the built-environ is required, for a key element of the urban terrain are the people engaging in flows of economic

\footnotetext{
${ }^{1}$ Note that FLWR identifies five environmental meta-trends; crowded, connected, lethal, collective, constrained.

${ }^{2}$ Kilcullen's networking encompasses at least FLWR's crowded and connected; See footnote 1.

${ }^{3}$ Of course, our deployed forces are then often in harms way.
} 
Is the Contested Urban Networked Littoral Environment a Step too far for Agent-Based-Modelling...

trade, and competing for resources within the built-environ.

This allows us to narrow our problem down. It is, how to model the human-terrain in order to support total ${ }^{4}$ integrated systems for future operations in the uncertain complex CUE?

\subsection{Simulation for Contested Urban Environments}

We have seen above the importance of the human element in the CUE, and human behaviours are often encapsulated as software agents ${ }^{5,6}$. To maintain its leading edge science and ensure it is positioned to take advantage of emerging technologies, DST Group has previously undertaken research across a wide range of scientific disciplines; as a small sample see (Millikan et al., 2011), (Johnson et al., 2011), (Ivancevic and Johnson, 2012), (Johnson and Ivancevic, 2013), (Ivancevic and Reid, 2014). This has supported Army in identifying and meeting its future requirements, with an emphasis on finding solutions to problems involving unconventional war and battle in uncertain ${ }^{7}$ human terrain and dynamic environments, and utilised computer simulations to computationally investigate population / crowd behaviour in complex environments.

\section{UNBOUNDED SYSTEMS THINKING}

We have recognised that the human terrain is identified by the military themselves and military pundits as being a dominant factor in dealing with violent conflict in CUE. Mitroff and Linstone (1993) attempt to break the chains of traditional business thinking by adopting an approach they call unbounded systems thinking ${ }^{8}$. They were motivated by recognising the flaws in old ways of thinking and deciding what to do next that were, at best, only suitable for a world that was and is no more. Now decision makers are dealing with the modern complex highly networked world in which we live. As part of the progression to the future of knowing, to see the world as an interconnected whole, they identify the historical development from old-thinking, through complex-thinking to new-thinking.

According to Mitroff and Linstone (1993) there are five ways of knowing or seeing the world, which are formally known as inquiry systems (IS). They are the following: a) agreement, an inductive IS (AIS); b) the world as a formula, an analytic IS (FIS); c) multiple realities, multiple model/data couplings or frames IS (MRIS); d) conflict, adversarial confrontation of thesis and antithesis IS (CIS) and finally e) unbounded systems thinking (UST). Mitroff and Linstone (1993) identify the first two with old-thinking, the next two with complex-thinking, and the fifth with new-thinking.

UST is a challenging paradigm to grasp. All of the simplicity of reductionism is torn down, leaving all in a rather uncomfortable position.
"We will find that in UST everything interacts with everything .... In UST, every one of the sciences and professions is considered fundamental; none is superior to or better than any other" (Mitroff and Linstone, 1993, $p$ 91).

UST moves beyond the MRIS manifold model/data couplings or frames to introduce multiple perspectives. In this concept agreement and analysis (AIS/FIS), are collectively subsumed into the Technical or T Perspective. The Multiple Perspective Concept UST involves three ways of dealing with complex problems. The $T$ Perspective is not replaced, but rather augmented with the $O$ or Organisational or societal perspective and the $P$ or Personal or individual perspective. $O$ and $P$ bring to the forefront human beings collectively and individually in all their complexity. The difference in perspectives forces us to discriminate between how we are looking from what we are looking at. Each perspective includes unique sets of underlying assumptions and values. For instance, applying T. O. P. perspectives to the concept of world-view reveals the paradigms

\footnotetext{
${ }^{4}$ Total integrated systems include hardware, software, people and business processes.

${ }^{5}$ Various agent models have been proposed to include the human element in two main ways; from a psychological perspective (Luger, 2002) or a sociological perspective (Gilbert and Troitzsch, 1999). The psychological emphasises an individual's cognition, while the sociological emphasises collective behaviour. Traditionally ABMs emphasises the sociological perspective (Gilbert and Troitzsch, 1999). ${ }^{6}$ Note though, Defence ABMs are weak on explicitly acknowledging what human science constructs they utilise (Johnson et al., 2011), being seen merely as tools for operational analysis. Defence constructive simulations, or ABMs, have been traditionally used as a force on force analysis tool for a variety of complex operational scenarios; see (Kish, 2011). They typically contain many entities, which can be thought of as agents. There are often a huge number of entities, whose behaviours can be programmed to represent any human behaviour, but have ususally been restricted to physical sensing and effecting, like sight and movement for instance.

${ }^{7}$ Current work which fundamentally addresses the issue of acting in an uncertain world can be found in Scholz and Reid (2015).

${ }^{8}$ They base this on an analysis of how people come to know anything at all, but have a very pragmatic approach to their management science and were strongly influenced by the thinking of $\mathrm{C}$. West Churchman, an American philosopher and systems scientist who was both a Professor of Business Administration and also Peace and Conflict Studies.
} 
Is the Contested Urban Networked Littoral Environment a Step too far for Agent-Based-Modelling...

of science-technology, social-entity and individuation/self, respectively, whereas applying it to the concept of goal gives problem-solving/product, action/stability/process and power/influence/prestige, respectively.

Following the UST of Mitroff and Linstone (1993) with regard to sociological aspects involved in studying military scenarios in the CUE our task is to apply T.O.P. perspectives: a) what are the technical issues? b) what are the organisational issues? and finally c) what are the personal issues? This is not restricted to just this complex problem though. It equally applies to ourselves as scientists in deciding what problems we should tackle and what methods we should use. It is extremely challenging to have the internal fortitude to apply UST to ourselves ${ }^{9}$. The reward, however is better alignment between our science, organisational and personal outcomes.

\section{SCIENTIFIC DISCIPLINES RELEVANT TO MODELLING THE HUMAN TERRAIN}

In this part of our horizon survey we attempt to identify some scientific disciplines or methods which may be of general use in modelling the human terrain. We commence with considering psychology as part of the $P$ perspective, but only briefly here ${ }^{10}$. We will spend more time on some of the basic aspects of sociology, $O$ perspective, not only because the interacting people appears to be crucial in CUE, but because we believe that it is an area poorly understood within defence modelling and simulation community ${ }^{11}$.

\subsection{Psychology}

We consider just two for our current purposes, which offer a psychological topology as an individual's model of the world (Lewin, 1997) and a simple and computationally efficient model of decision making (Hertwig and Herzog, 2009).

Lewin's (1997) topological approach is quite general and abstract and may be formulated in a number of ways to suit the problem at hand. In brief (Johnson and Ivancevic, 2013) summarises the nine constructs of Lewin's field-theory as follows: 1. Position for location and the related geometry of relative position (i.e., topology); 2. Locomotion or relation of geometric position over time; 3. Cognitive structure for the relative position of various geometrical objects (points, boundaries, regions, etc.); 4. Force for the tendency to locomotion and change over time, and the accompanying force-field for the spatial distribution of forces; 5 . Goal for a forcefield focused on a particular point, with a positive valence; aversion is similar but with a negative valence; 6 . Conflict $^{12}$ for at least two overlapping competing force-fields, which may result in a vacillatory equilibrium for certain constellations of overlapping force-fields; 7. Fear which is related to the psychological-future; 8. Power which refers to a "possibility of inducing forces" on another person via a power-field, e.g., a parent's power over a child; 9. Values refers to internal or self regulating power ${ }^{13}$.

In contrast Hertwig and Herzog (2009) have another view from within psychology, emphasising the importance of the irrational, yet highly effective, fast and frugal heuristics that humans use to make decisions within the context of bounded-rationality ${ }^{14}$. Two examples of fast and frugal heuristics should suffice to illustrate the idea; the recognition heuristic, and the take the best heuristic. In the recognition heuristic when a choice between alternatives is presented, if only one is recognised, then choose it. In contrast, in the take the best heuristic, when searching for a solution take the best alternative, but only based on the first cue that discriminates between the alternatives. Both are clearly irrational, however the time taken to decide is quick and not much effort is required to do so. Perhaps people ought not do this from a purely logical perspective, but according to Hertwig and Herzog (2009) this is what people often actually do. Interestingly, in the context of ABM in addition to basing decision making on a known psychological construct, this also eases the computational load on our simulation systems, because of the frugal nature of this method of decision making.

\footnotetext{
${ }^{9}$ This is not just some cute academic point said in passing. We as scientists are not on the edge of the world staring in at the problem, we are always part of it from the UST point of view. "The systems designer, analyst, or manager is a fundamental part of the system or problem being analysed; that individual's psychology as well as social context are inseparable from how he or she represents a system or problem" (Mitroff and Linstone, 1993, p99).

${ }^{10}$ See (Johnson and Ivancevic, 2013), (Johnson and Ivancevic, 2015) and references therein for a fuller survey.

${ }^{11}$ We will omit the $T$ perspective since in the defence community it is normally covered in great detail and we will instead focus here on what is commonly missed; the $O$ and $P$ perspectives.

${ }^{12}$ This refers to psychological conflict within the life-space, not violence in a physical space.

${ }^{13}$ Although Lewin's life-space is intended to model the psycho-biological-space of an individual, if that individual is in a mode where social identity is salient, then it is easy enough to see how it can be extended to a social-group-space. For a full explanation of these concepts see (Johnson and Ivancevic, 2015). Further, Ivancevic and Johnson (2012) indicates how in principle Lewinian principles may be applied to problems concerning social groups and societies.

${ }^{14}$ From an evolutionary perspective this tendency may have evolved because of the speed at which creatures need to take decisions in order to survive.
} 
Is the Contested Urban Networked Littoral Environment a Step too far for Agent-Based-Modelling...

\subsection{Sociology}

Sociological theory according to Macionis and Gerber (2010) consists of seven components. They are: culture, society, socialisation, social interaction in everyday life, groups and organisations, sexuality and society and finally deviance. We merely introduce the concepts as things that ought to be considered in modelling the human terrain, and expand on the some of the more relevant aspects. There is no demand to use all of them. At this stage we seek merely to create a mutual understanding between the various scientific disciplines.

With respect to culture it is necessary to understand it in terms of, firstly, a way of life and, secondly, the elements of culture per se. The way of life is a construct of what people do and what people have, which has two aspects, the material, meaning physical things or material symbols, and the non-material, meaning non-material symbols, such as ideas, language, values, beliefs and norms that are the elements of the culture. Symbols refers to anything carrying meaning, while language is considered as a system of symbol manipulation and of course, for interpersonal communication. Values are the defined standards for the how good something is, and beliefs are statements or assumptions that people hold to be true, while norms are the rules of expected behaviour. Norms consist of mores, which are widely observed standards in the society and are held to be of great moral significance, where folkways, by contrast, are standards for routine casual interaction.

A society then is defined as people who interact in a defined territory and share a culture. Traditionally, of course a territory refers to a geographic region which has defined physical boundaries. In the social sciences it is an aphorism to say that "physical boundaries create social boundaries". Within a society the process of socialisation refers to life long learning through social experience that contributes to the development of individual personality and allows full participation in that particular society. Social interaction in everyday life refers to the "rules" that guide behaviour in everyday situations and is the process by which people act and react in relation to others, which includes both emotions and language. Groups and organisations are important sociological terms to understand. A social group has two or more people who interact and identify with one another ${ }^{15}$.

Deviance refers to the state of standing out by not conforming to what is expected behaviour or a norm and is a reflection of both individual choice and the norms and the conventional operation of a particular society. It is the recognised violation of social rules and cultural norms. Norms guide almost all human activities, ranging from minor infractions, such as bad manners, to major ones, like serious violence.

\section{A SMALL EXAMPLE CASE STUDY}

We have briefly covered some areas, in isolation, in section 3 that might be generally useful in modelling the human terrain. Now motivated by the need to bridge between the sociological and psychological perspectives, we present here one small example case study, on some issues in integrating models of cognitive behaviour and sociological behaviour within ABMs

As intimated previously, normally within ABMs cognition is ignored (Gilbert and Troitzsch, 1999). Gilbert (2006) has later commenced a discussion on this issue in isolation. There has been much research reported in the psychology literature on theories of mind, learning and development and on Cognitivism in general, for the individual ${ }^{16}$. We have already seen above, that in the area of social sciences, theories concern themselves largely with the interactions between actors in a society. Additionally, but not previously discussed here, there is also a large body of literature on distributed artificial intelligence (DAI) from those seeking to simulate the behaviour of individuals, or groups, or both ${ }^{16}$. One problem is that this literature exists in three separate and traditionally distinct groups of disciplines, which must be drawn upon in order to produce viable and plausible executable models. Despite this, a general understanding of these three discipline groups is still required. We now unpack in a little more detail just which disciplines need to be considered ${ }^{16}$. Firstly, psychology and cognitive sciences, e.g. (Hertwig and Herzog, 2009); secondly, the social sciences including behavioural economics and politics, e.g. (Gilbert and Troitzsch, 1999); and finally, DAI, agents, and cybernetics, e.g. (Luger, 2002).

Clearly such a wide range of relevant scientific disciplines creates a substantial challenge for integration, and will require careful application of the UST approach of Mitroff and Linstone (1993).

\footnotetext{
${ }^{15}$ The group size may small and personal or large and impersonal and will act according to some sort of general rules. A formal organisation is a large secondary group organized to efficiently achieve its goals, like a rationally desinged bureaucracy. Formal organisations are utilitarian, normative and coercive. With respect to intragroup and intergroup contact, it is generally true that large groups turn inward, while heterogeneous groups turn outward in their interactions and that, of course, physical boundaries create social boundaries.

${ }^{16}$ For a fuller summary see (Johnson and Ivancevic, 2015).
} 
Is the Contested Urban Networked Littoral Environment a Step too far for Agent-Based-Modelling...

\section{DISCUSSION AND CONCLUSION}

In one sense the purpose of this paper is to start a conversation. We anticipate that under UST, we will have to move considerably past old-thinking, into complex-thinking at a minimum, to end in debate, which Mitroff and Linstone (1993) denote as conflict. As an organisation ${ }^{17}$ we expect to have to practice new-thinking, UST, in order to reach organisational goals despite anticipated technical difficulties.

Kilcullen (2014) has illustrated through the mobile phone example how the human-terrain, translates to a turbulent sea of population movements towards littoral mega-cities, while Military pundits and the Military themselves have emphasised the importance of the transmission of idea-systems, in particular, and the human element in general. Two psychological constructs have been selected as promising ways to either represent knowledge or implement computationally efficient decision making schemes within ABMs (Lewin, 1997), (Hertwig and Herzog, 2009).

The UST and sociological aspects of the horizon survey can be summarised with some uncomfortable comments on the importance of modelling mature sociological interactions or communications. We can take mature to mean fully developed or having reached the most advanced stage in a process, while communication can be taken to mean the imparting or exchanging of information by utterances/speaking, markings/writing or using some other medium such as a creature's/human's-face and or body.

There are a progression and regression of comfortable and uncomfortable communication events in life whose occurrence and timing have deep elements of chance and uncertainty ${ }^{18}$. As part of problem solving we search for meaning in the world as we see it, and the meanings we discover or attribute to things and other creatures and people in our world are constructed for us and by us ${ }^{19}$. As social beings we mirror or copy each others constructed meanings and pass these on verbally and non-verbally, and once again sometimes we mirror because it's useful to save us the cost in time of figuring it out for ourselves. Sometimes we mirror because someone, a particular social group or social institution, has social power over us. What is clear is at a bare bones minimum, we must consider idea systems and information transmission (social interactions) in the context of factions (social groups) from a sociological perspective, in addition to our usual military comms T perspective. In modelling communications between agents within the ABM we must recognise these could be motivated by either individual psychological forces, or collective pressure from social groups and/or societal institutions.

In a competitive contested uncertain complex dynamic world, we are fundamentally functional, constructivist, meaning-making beings, attempting to participate in mature communication within and between our social groups and societies ${ }^{20}$. To successfully model these processes, we need to carefully ensure we do not pass judgment on the worth or otherwise of these meaning-making constructions. This enables us to view any agent as a being in a life flow process; for ultimately we all are instances of a human being (a process view; a flow), not a human be (a state space view; a thing). This is the social world that we all live in.

Section 4 also illustrates problems between scientific-disciplines in integrating sociological and psychological perspectives within our ABMs and constructive simulations. From an UST frame, in the business of acting as scientists in a multi-disciplinary teams, we must admit that the view of each discipline is worthwhile, and none subsumes the other. In each problem, we must strive for the following: a) balance among $T, O$ and $P$ perspectives; b) use "good" judgment in selecting perspectives; c) in obtaining information, recognise that $O$ and $P$ require different methods than $T ; \mathrm{d}$ ) pay particular attention to the mutual impact, interdependencies, and integration of perspectives; e) beware of thinking statically in dynamic environments.

So then, is the Contested Urban Networked Littoral Environment a Step too far for Agent-Based-Modelling? In short, answering from the T. O. P. perspectives: yes; perhaps; no. Though perhaps we only have some uncomfortable questions. From a $T$ perspective; are our own existing constructive simulations up to the task, given that they were conceived of and decided with old-thinking in mind, a 1970s frame of mind? From a $O$ perspective: are we properly skilled? are our tools sharpened enough for the task? can we form appropriate functioning listening multi-disciplinary teams? and crucially can we afford the capability decision risk of having no model at all that indicates the broad trends that ABMs are renown to give? From a P perspective: are we up for it?

\footnotetext{
${ }^{17}$ Organisation is used here in a social science sense and not as our label or name.

${ }^{18} \mathrm{In}$ an evolutionary sense we have grown to become excellent problem solvers and communicators, because it has enhanced the success of us as individuals, kinship groups, social communities, societies and as a species.

${ }^{19}$ Socially constructed means that we just made it up; it is socially or if you like culturally defined. Sometimes this is useful to us individually and sometimes it's not.

${ }^{20}$ We take functional to mean humans are task oriented problem solvers and communicators, while constructivist means we just make things up according to our psychological or social world views, in order to make meaning of our world.
} 
Is the Contested Urban Networked Littoral Environment a Step too far for Agent-Based-Modelling...

So here we stand looking at the T. O. P. perspectives, at the bottom of our own Mount Everest, with little more than Ugg boots and wet tee-shirts. It is a climb of tremendous difficulty and yet it is also of monumental importance if we are ever to study the all important violent conflict in dynamic CUE. This is not just a little wake up call. We have issued the call to arms, made that dreaded clarion call, sent out the invitations, rung that blooming bell hard, not because we can, not because we want to, but because we must.

\section{REFERENCES}

Clarke, R. A. (2004). Against all enemies: inside America's war on terror. New York: Free Press.

Day, S. (2015, June). Thoughts on generalship: Lessons from two wars. ARP 5.

Gilbert, N. (2006). When Does Social Simulation Need Cognitive Models? In R. Sun (Ed.), Cognition and multi-agent interaction: from cognitive modeling to social simulation, pp. 428-432. Cambridge: Cambridge University Press.

Gilbert, N. and K. G. Troitzsch (1999). Simulation for the Social Scientist. Buckingham: Open University Press.

Hertwig, R. and S. M. Herzog (2009). Fast and frugal heuristics: tools of social rationality. Social Cognition 27(5), 661-698.

Ivancevic, V. G. and W. T. Johnson (2012). Macro-microscopic self-similarity in socio-cognio-physical dynamics. Journal | Mathematics in Engineering, Science and Aerospace 3(3), 325-339.

Ivancevic, V. G. and D. J. Reid (2014, December). Complexity and Control: Towards a Rigorous Behavioral Theory of Complex Dynamical Systems, Volume 20 of Series on Stability, Vibration and Control of Systems, Series A. WORLD SCIENTIFIC.

Johnson, W. T. and V. G. Ivancevic (2013, December). Individual Psychological Conflict within Social Group Dynamics. In J. Piantadosi, R. Anderssen, and J. Boland (Eds.), Proceedings of MODSIM2013, Adelaide, Australia, pp. 1026-1032. Modelling and Simulation Society of Australia and New Zealand.

Johnson, W. T. and V. G. Ivancevic (2015). Progress Toward a Psycho-Physical Holistic Embodied Model of Being. DST-Group-TR (to appear), DST Group, Edinburgh, Australia.

Johnson, W. T., M. Wong, and P. Jacques (2011, July). A study of issues involved in using an explicit cognitive model within an Agent-Based-Model crowd scenario. In Invited Presentation Session on Holistic Approaches to Military and Security Modelling at IFORS 2011, Melbourne, Australia.

Kilcullen, D. (2014, August). The Australian Army in the Urban Networked Littoral. ARP 2.

Kish, A. (2011, June). COMBATXXI: Usage and Analysis at TACOM. TACOM-Report 21845, US Army RDECOM-TARDEC.

Lewin, K. (1997). Resolving Social Conflicts and Field Theory in Social Science. Washington, DC.: American Psychological Association.

Luger, G. F. (2002). Artificial intelligence: structures and strategies for complex problem solving (fourth ed.). Harlow: Addison-Wesley.

Macionis, J. J. and L. M. Gerber (2010, January). Sociology (7 ed.). Pearson Education Canada.

Millikan, J., D. Grieger, and W. J. Chau (2011, July). From Conceptual to Holistic Modelling: Societal Responses of Populations in Crisis using an Opinion Dynamics Model. In Invited Presentation Session on Holistic Approaches to Military and Security Modelling at IFORS 2011, Melbourne, Australia.

Mitroff, I. I. and H. A. Linstone (1993). The unbounded mind: breaking the chains of traditional business thinking. New York: Oxford University Press.

MSPD-Staff (2014, April). Future Land Warfare Report. FLWR 2014, Australian Army Headquarters, Modernisation and Strategic Planning Division.

Scholz, J. B. and D. J. Reid (2015). So, where are all the robots? A rationale for strategic research (available to qualified readers). DST-Group-RR 0420, Edinburgh, Sth. Aust.

Sengelman, J. (2014, May). Delivering the Future. In Brief to DSTO, Edinburgh, Adelaide, Australia. 